\title{
LEVELNET: DIVING INTO THE MULTIPLE LAYERS OF PROTEIN-PROTEIN PHYSICAL INTERACTION NETWORKS
}

\author{
Yasser Mohseni Behbahani \\ Paul Saighi \\ Flavia Corsi \\ Elodie Laine* \\ Sorbonne Université, CNRS, IBPS \\ Laboratory of Computational and Quantitative Biology (LCQB), UMR 7238 \\ Paris, 75005, France \\ * Corresponding authors: \\ alessandra.carbone@sorbonne-universite.fr \\ elodie.laine@sorbonne-universite.fr
}

Alessandra Carbone*

July 31,2021

\begin{abstract}
The complexity underlying protein-protein interaction (PPI) networks calls for the development of comprehensive knowledge bases organizing PPI-related data. The constant growth and high reliability of structural data make them a suitable source of evidence for the determination of PPI. We present LEVELNET, a fully-automated and scalable environment designed to integrate, explore, and infer protein interactions and non-interactions based on physical contacts and other PPI sources, including user-defined annotations. LEVELNET helps to break down the complexity of PPI networks by representing them as multi-layered graphs and allowing the selection of subnetworks and their direct comparison. LEVELNET proposes an interactive visualisation based on a user-friendly web interface. LEVELNET applications are multiple. It allows to explore PPIs of biological processes, identify co-localised partners, assess PPI predictions from computational or experimental sources, unravel cross-interactions, show and compare multiple PPI sources, and help creating PPI benchmarks with specific properties.
\end{abstract}

Availability: LEVELNET is freely available to the community at http://www.lcqb.upmc.fr/levelnet//

\section{Introduction}

Most of the cellular machinery and biological processes are fulfilled by biomolecular associations, particularly proteinprotein interactions (PPI). The versatile, adaptable, and specific nature of proteins gives them the ability to create networks that govern virtually all intra- and inter-cellular activities. Analyzing these networks enlightens the relative importance of proteins in different organisms and communities. It improves our understanding of physiopathological mechanisms and helps us to decipher gene-disease-drug associations and find therapeutic treatments $(25,33,30,13$, 37). The ever increasing growth of protein sequential, structural, and functional information $(39,38,3,10)$ and of experimental evidence for PPI (27, 26, 29, 28, 14) has stimulated the development of databases and web-interfaces for curating, inferring, and browsing PPI networks. These resources compile experimental and computational data and provide user-interactive visualization. Moreover, since PPI-related data may be contradictory, noisy, heterogeneous, and biased (11, 15), a lot of effort has been invested to integrate other types of information (e.g. cellular localisation), 
increase the interpretability, organize the PPI based on their biological context, and compute confidence scores. These characteristics are implemented in IID (15), STRING (36, 34, 35), and the human-focused base HIPPIE (2).

Graphs are convenient and compact data representations for biological processes. Ideally, graphs representing PPI networks should reflect their complexity as information-rich systems where each node might contribute to the whole network more than just to a part. A PPI is not a simple edge between two nodes but can be viewed as a multi-edge, where the multiplicity comes from the different sources of evidence, associated with a set of weights reflecting either a property from the source or the reliability of the evidence. Graphs formed upon diverse sources may explain the existence of multiple interaction sites on the surface of a protein (18, 8). Additionally, it is desirable that such graphs encode information about non-interacting pairs.

While most of the databases describe interactions without accounting for any underlying physical information (5, 16, 9, 12, 20), a handful of them build upon protein 3D structural evidence, e.g. Interactome3D (23), PPI3D (6), and 3 did (24). Structural determination techniques provide experimental biologists with atomic-level resolution about the formation of protein complexes. This information is also useful to assess docking algorithms and generate binary- and cross-interaction benchmarks. Moreover, under the hypothesis that some PPI are conserved in evolution one may infer the existence of unseen interactions by transferring knowledge from homology.

Here, we report on LEVELNET, a fully-automated and scalable PPI knowledge base coupled with a user-friendly web-interface designed to integrate and explore PPI networks coming from multiple sources. LEVELNET exploits physical contacts and homology relationships to build multi-layered PPI networks. It allows cross-examining these networks through the extraction and comparison of a multitude of layers, each one corresponding to a source and a confidence level. Moreover, it operates on a hierarchy of biological entities defined from sequence similarities. (Figure 11. It primarily relies on structural information coming from the Protein Data Bank (PDB), and also integrates positive and negative information on pairwise interactions from HIPPIE and Negatome (31) respectively. Beyond the immediate value for exploring whether and how a group of proteins establishes physical contacts, LEVELNET proved useful to assess predicted outcomes or compile benchmark sets for training and testing predictive methods on PPI-related tasks.

\section{Materials and methods}

\subsection{Input}

LEVELNET takes as input a set of proteins or protein chains (PDB identifiers) possibly accompanied by a matrix specifying the existence and strength of some relationships between them (Figure $1 \mathrm{~A}$ ). The matrix should be given as a list of triplets: protein1, protein2, and associated score (i.e., a value between 0 and 1; if a score is missing, the value 1 is taken).

\subsection{Multi-layered PPI network representation}

LEVELNET compiles PPI networks from several sources of evidence in a multi-layered structure (Figure 11B) and acts, on each layer, on both nodes and edges. It annotates edges by sequence similarity levels (in case of PDB), confidence scores, or any type of user-defined annotations. By diving into the different layers, one can readily appreciate the rewiring of LEVELNET PPI networks upon relaxing the edge threshold. This feature allows for the selection and the visual comparison of different PPI layers defined based on confidence scores and multiple sources (Figure 1 1 C). To ease visualization, LEVELNET spatially arranges the nodes according to the topology of the network.

Redundancy reduction. LEVELNET can create a non-redundant version of the network. For this, it defines super-nodes as groups of homologous input chains (Figure $1 \mathrm{~F}$ ) that belong to pre-computed clusters of sequence identity available from RCSB PDB (computed using MMSeqs2; 32). It defines an edge between two super-nodes, whenever there is at least a pair of (non-)interacting chains between them. Multiple edges between super-nodes will correspond to different sources of evidence.

LEVELNET allows for the selection of subnetworks delineated with respect to a node or a connected component: 
bioRxiv preprint doi: https://doi.org/10.1101/2021.07.31.453756; this version posted August 2, 2021. The copyright holder for this preprint (which was not certified by peer review) is the author/funder, who has granted bioRxiv a license to display the preprint in perpetuity. It is made available under aCC-BY-NC-ND 4.0 International license.

\section{LEVELNET: DIVING INTO THE MULTIPLE LAYERS OF PROTEIN-PROTEIN PHYSICAL INTERACTION NETWORKS}
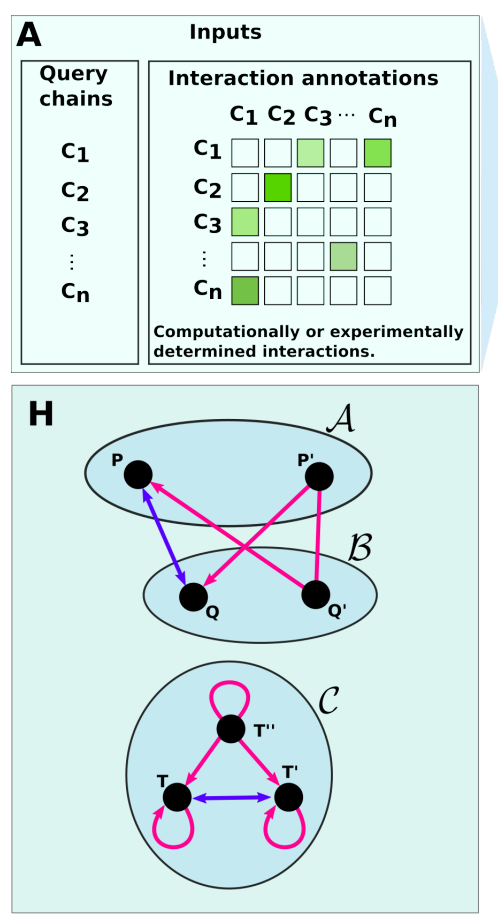

Input chain

Selected chain

Chain in the same complex
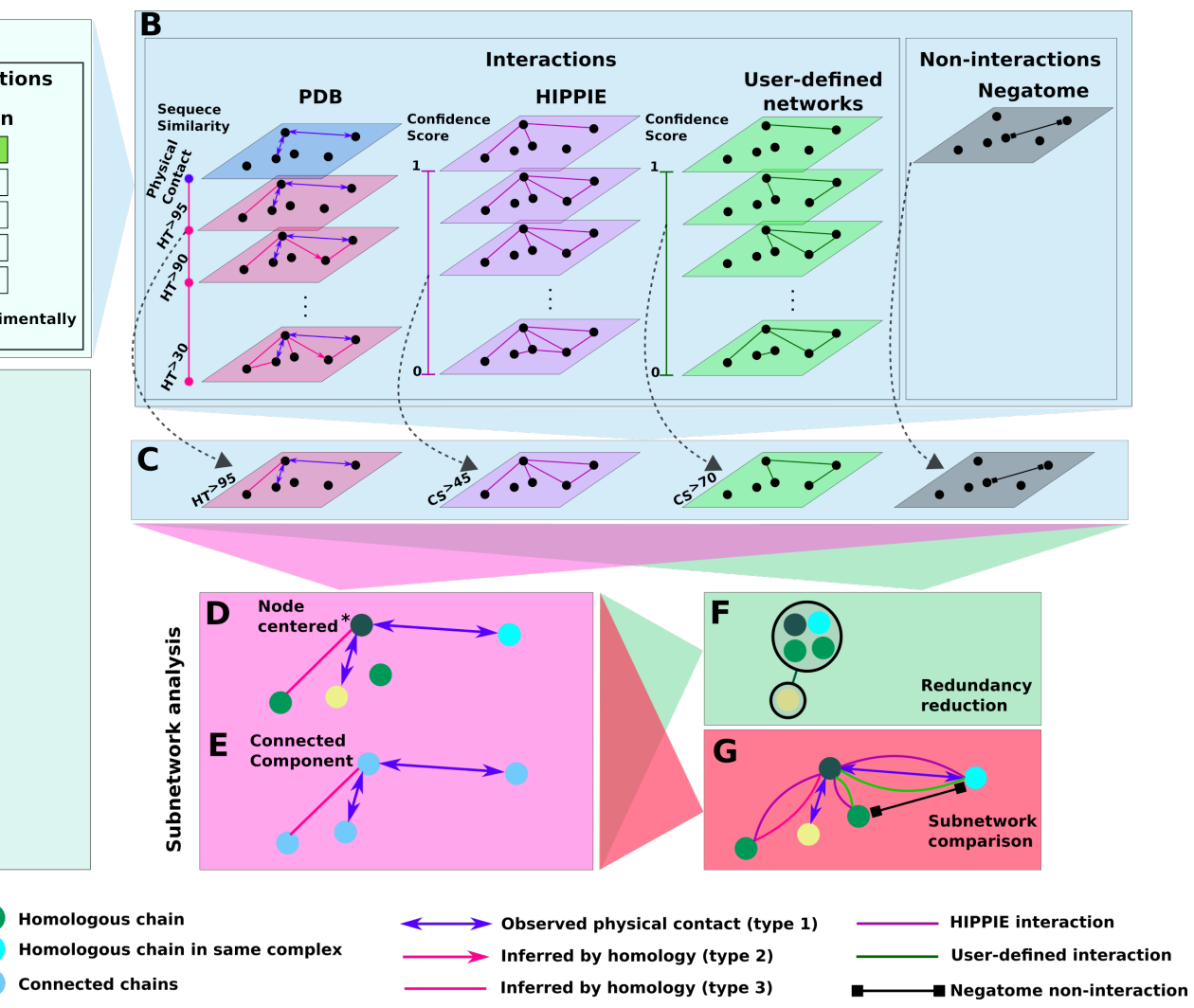

Figure 1: Multi-layered representation and analysis of the PPI networks in LEVELNET. (A) Two kinds of inputs are possible, either a list of proteins (or protein chains) or a matrix of annotated interactions. (B) Multi-layered networks compilation for the given query from the database. (C) Visual comparison in different levels of confidence and across various sources. (D) Node-centered subnetwork selection (the central node is shown by *). (E) Connected component subnetwork selection. (F) Non-redundant representation of a selected (sub)network with super-nodes (black circles) representing groups of homologous chains. (G) Aggregated representation of the subnetwork. (H) LEVELNET infers three types of interactions using homology transfer. $\mathcal{A}, \mathcal{B}$, and $\mathcal{C}$ are clusters at a fixed level of sequence similarity, containing chains $\mathrm{P}$ and $\mathrm{P}^{\prime}, \mathrm{Q}$ and $\mathrm{Q}^{\prime}, \mathrm{T}, \mathrm{T}^{\prime}$ and $\mathrm{T}^{\prime \prime}$, respectively. Chains $\mathrm{P}$ and $\mathrm{Q}$ create an inter-cluster contact between $\mathcal{A}$ and $\mathcal{B}$ due to their interaction of type 1, therefore all chains of $\mathcal{A}$ cross-interact with all chains of $\mathcal{B}$ at type 3 level and all chains of $\mathcal{A}(\mathcal{B})$ cross-interact with $\mathrm{Q}(\mathrm{P})$ at type 2 level. Chains $\mathrm{T}$ and T' create an intra-cluster contact in $\mathcal{C}$ due to their interaction of type 1 and cause the creation of both self- and cross-interactions for all chains of the cluster at type 1 and 2 levels.

- Node-centered selection highlights chains of the same complex and its homologs (belonging or not to the complex) and analyze how they interact with the rest of the network (Figure 1D). This functionality helps, for instance, to detect homo-oligomers.

- Connected component selection gives access to groups of proteins that directly or indirectly interact together, as in a signaling pathway for instance (Figure $1 \mathrm{E})$.

Once the user has selected a subnetwork, s/he can perform subnetwork comparison and create a multi-edge graph by integrating information coming from different sources, and directly compare the interactions (Figure $1 \mathrm{G}$ ) by visualising their superimposition.

\subsection{Types of (non-)interactions and inference algorithm}

Relations between protein chains are represented by six types of edges (Figure $1 \mathrm{~B}$ ):

- type 1: observed interaction, i.e. the two chains show a physical contact in a known complex 


\section{LEVELNET: DIVING INTO THE MULTIPLE LAYERS OF PROTEIN-PROTEIN PHYSICAL INTERACTION NETWORKS}

- type 2: interaction inferred by homology where one of the chains is in physical contact with a homolog of the other chain

- type 3: interaction inferred by homology where some homologs of the two chains are in physical contact

- type 4: HIPPIE annotated interaction

- type 5: NEGATOME annotated non-interaction

- type 6: User-defined interaction

The absence of an edge indicates missing supporting evidence for an interaction or a non-interaction.

LEVELNET infers the three first types of edges directly from the PDB ("PDB" source in Figure 1B). To achieve computational and memory efficiency, we pre-computed two databases, one of physical interfaces, and one of physical and homology-transferred interactions:

Database of physical interfaces. We first downloaded all PDB entries from the June 2020 release, and identified the residues at the interface between every pair of protein chains in the same biological assembly (from X-ray crystallography and cryogenic electron microscopy) or NMR model using INTBuilder (7). PDB entries with more than 100 chains or with a resolution lower than $5 \AA$ were discarded. Protein chains smaller than 20 residues or with more than $20 \%$ of unknown residues were also discarded. Two residues were considered as in contact if the distance between any of their atoms was less than $5 \AA$.

Database of physical and homology-transferred interactions. We generated an optimized database of interactions containing all necessary information for inferring type 1, type 2, and type 3 edges. We determined the set of observed physical contacts (type 1: chain-chain interactions) by looping over all possible pairs of chains in PDB complexes and verifying the existence of a contact in our previously computed database of physical interfaces. Then, we transferred interaction knowledge between homologous chains (types 2 and 3) (Figure $1 \mathrm{H}$ ). For this, we exploited again the pre-computed data structure of RCSB PDB organizing the PDB chains into sets of clusters defined at 30, 50, 70, 90 and 95\% sequence identity. That is, if

$$
\exists P \in \mathcal{A} \exists Q \in \mathcal{B} . P \neq Q \wedge|\{(r, s) \mid r \in P, s \in Q, d(r, s)<D\}|>M
$$

then the following edges are inferred in LEVELNET:

- $P \longleftrightarrow Q$ (type 1: this connection is shown as a double-arrow blue edge)

- for all $P^{\prime} \in \mathcal{A}$ and $Q^{\prime} \in \mathcal{B} . P \neq P^{\prime}$ and $Q \neq Q^{\prime}$

- $Q^{\prime} \longrightarrow P$ (type 2 : pink directed edge)

- $P^{\prime} \longrightarrow Q$ (type 2: pink directed edge)

- $P^{\prime}-Q^{\prime}$ (type 3: pink undirected edge)

where $\mathcal{A}$ and $\mathcal{B}$ are two clusters of chains in a certain percentage of sequence identity (Figure $1 \mathrm{H}$ ), $d(r, s)$ defines the distance between two residues $r$ and $s, D$ is the distance threshold set to $5 \AA$ by default, $M$ is the minimum number of interface residues set to 5 for both proteins by default. Note that the process above also allows describing the relations of the chains within a cluster (Figure $1 \mathrm{H}$, see cluster $\mathcal{C}$ ).

To perform homology transfer, we implemented a fast routine that necessitates to loop only over the identified directly interacting chain pairs $(P, Q)$ and determine sets of chain-cluster and cluster-cluster interactions, for each level of sequence identity considered:

1. foreach pair $(P, Q)$ do

2. $\quad \mathcal{A} \leftarrow$ find_cluster $(P)$

3. $\quad \mathcal{B} \leftarrow$ find_cluster $(Q)$

4. P.set_cluster $(\mathcal{A})$

5. Q.set_cluster $(\mathcal{B})$

6. $\mathcal{A}$.add_neighbour $(\mathcal{B})$

7. $\mathcal{B}$.add_neighbour $(\mathcal{A})$

8. $\quad$.add_target $(\mathcal{B})$

9. $Q . \operatorname{add} \_\operatorname{target}(\mathcal{A})$

10 . end 
Lines 2 and 3 find the clusters to which chains $P$ and $Q$ belong at a certain percentage of sequence identity and lines 4 and 5 set the cluster identifiers as their properties. On lines 6 and 7, the function add_neighbour sets a cluster-cluster interaction. As a result, each cluster will have a set of neighbours with which it interacts in an undirected way. On lines 8 and 9, the function add_target sets two chain-cluster interactions. These interactions are directed from the chain to the cluster.

\section{Processing an input query}

Starting from the input query, LEVELNET will interrogate the pre-computed database of physical and homologytransferred interactions to create type 1,2 and 3 edges. For each chain pair in the query, if there is a chain-chain interaction, LEVELNET creates a type 1 edge. If not, then if there is a chain-cluster interaction it creates a type 2 edge, otherwise if there is a cluster-cluster interaction, it creates a type 3 edge. LEVELNET will also interrogate HIPPIE and Negatome to infer type 4 and 5 edges. Finally, if the user specified some relationships between the input chains, LEVELNET will integrate them in the output multi-layered network as type 6 edges.

\subsection{LEVELNET implementation details}

To generate the PDB interaction database, we developed a pipeline in Python that processes PDB protein complexes, their interfaces, and chain clusters by sequence identity. We created an interactive environment based on recent advances in web development including HTML5 and related technologies such as D3, JavaScript, Vue, and SVG. HTML5, CSS3 and Vue are used for the front-end and provide a stylish and user-friendly interactive interface. Data visualization is performed using D3 (4) and SVG. Codes developed in JavaScript and Python process databases and user queries. The whole pipeline is optimized to respond to queries rapidly.

\subsection{Benchmark creation}

To create a new benchmark set, we first performed an advanced search in the PDB. The criteria for this search were the following: protein as Polymer Entity Type, Hetero 3-mer as Oligomeric State, Polymer Entity Sequence Length between 100 and 300, Refinement Resolution better than $2.5 \AA$. Moreover, all complexes related to antibody-antigen (e.g. FAB) were excluded.

\section{Results and discussion}

\subsection{Exploring a biological process}

We used LEVELNET to explore the interactions at play in the Calvin-Benson cycle, a series of biochemical redox reactions taking place in photosynthetic organisms. We considered the green alga Chlamydomonas reinhardtii as an example. From 10 input PDB complexes, LEVELNET inferred 1430 pairwise interactions between 108 protein chains at $95 \%$ sequence identity (Figure 2). The denser connected component links the chains coming from the Ribulose-1,5-bisphosphate carboxylase-oxygenase (RuBiSCo) complexes 1GK8, 1UZD, and 1IR2 (Figure 2A). Each complex comprises several copies of a small ( $\mathrm{S}$, in red) and a large ( $\mathrm{L}$, in blue) subunits, which appear as supernodes in the non-redundant network. The other two connected components (Figure 2B,C) involve photosystem light harvesting complexes of types I (6JO5 and 6JO6) and II (6KAC). The remaining subnetwork (Figure2D) displays some inconsistencies between the PDB and the Negatome (Figure 2F). Specifically, the chain pairs ML, MG, MN, DB, DC, and DG from 1Q90 (a cytochrome b6f enzyme) are annotated as non-interacting in the Negatome while they are found in physical contact in the PDB (Figure $2 \mathrm{G}$ ). Following redundancy reduction at 95\% similarity, LEVELNET inferred 98 pairwise interactions between 55 super-nodes.

\subsection{Accounting for protein co-localization}

Next, we focused on the canonical Wnt signaling pathway, which regulates gene transcription by passing signals from the cell surface receptors to the nucleus. A large number of X-ray, NMR and cryoEM structures characterizing this pathway are available and were recently reviewed in (1). The authors provide a list of 85 PDB codes organized according to their cellular localization and role: secreted positive or negative regulators, transmembrane proteins, cytoplasmic proteins and intranuclear proteins. Starting from the list and the co-localization annotations, LEVELNET built a network comprising 235 nodes linked by 2734 edges inferred from the PDB and by homology transfer above $30 \%$ sequence identity. By spatially arranging the nodes according to their co-localization, LEVELNET gives an overview of the full pathway, where proteins sharing the same location and role are grouped together and where interactions between groups are clearly distinguishable (Figure 33. Upon merging the nodes sharing more than $30 \%$ sequence identity, the 
bioRxiv preprint doi: https://doi.org/10.1101/2021.07.31.453756; this version posted August 2, 2021. The copyright holder for this preprint (which was not certified by peer review) is the author/funder, who has granted bioRxiv a license to display the preprint in perpetuity. It is made available under aCC-BY-NC-ND 4.0 International license.

LEVELNET: DIVING INTO THE MULTIPLE LAYERS OF PROTEIN-PROTEIN PHYSICAL INTERACTION NETWORKS
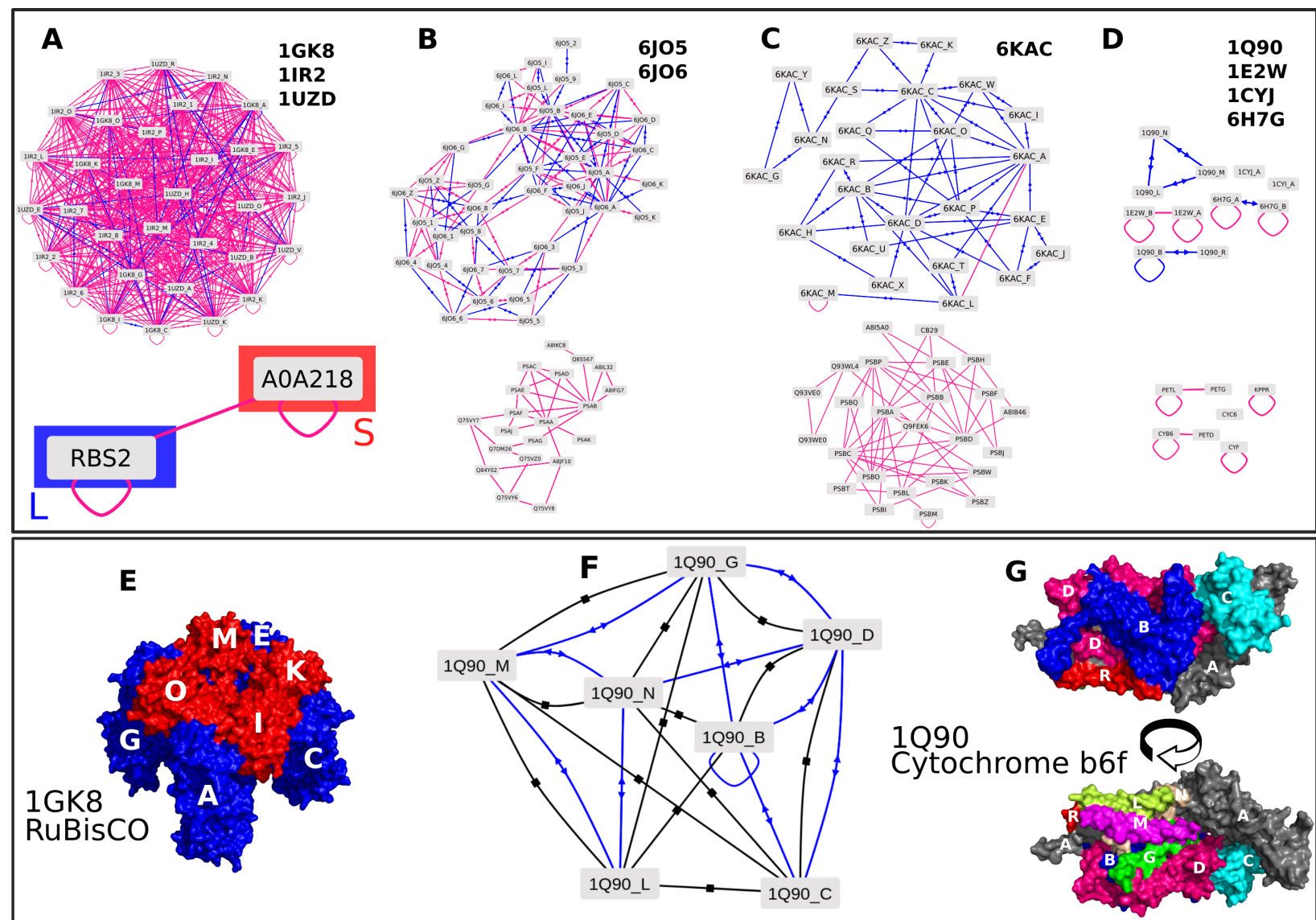

Figure 2: Physical PPI network for the Calvin-Benson cycle of Chlamydomonas reinhardtii. (A-D) Main subnetworks inferred from the PDB at $95 \%$ sequence identity. The input PDB codes are indicated in the top right corners. In each panel, the layer shown on top has one node per structural chain. In the layer below, the nodes sharing more than 95\% sequence similarity have been merged. The connected components correspond to the RuBiSCo complex (A), and the photosystem light harvesting complexes of types I (B) and II (C). (D) Other protein chains forming small networks. (E) For the RuBiSCo complex, we also show its 3D structure (PDB code: 1GK8), with the small and large subunits in red and blue respectively. (F) Subnetwork comparison of two layers, corresponding to observed interaction in the PDB (blue) and non-interactions from Negatome (in black) for the cytochrome b6f enzyme (1Q90). (G) Two views of the structure 1Q90.

size of the network reduces dramatically down to less than 50 nodes. This non-redundant version allows reasoning at the protein level, toward identifying the parts of the pathway that are described by structural information and the parts where such information is missing (Figure 3 compare the inset with Figure 1 from (1)). Several complexes formed in the membrane are clearly identifiable, for instance between Dickkopfs (DKKs), Kremen (KREM1) and LRP6, between R-spondins (RSPOs) and LGR family receptors, and between Wnt proteins (WNTs) and frizzled receptors (FZDs) (Figure 3 grey panel in inset, from left to right). $\beta$-catenin (CTNB1) appears as a crucial protein in the pathway, with multiple interactions with cytoplasmic proteins (green panel) and with intranuclear proteins (blue panel). In particular, in the cytoplasm (green panel), its interactions with axin (AXN) and Adenomatous Polyposis Coli (APC) contributing to the formation of the degradosome were structurally characterized. So were its interactions with the scaffold protein BCL9/legless, Groucho/TLE, and $\beta$-catenin binding proteins/domains (CNBP1, TCF3-CBD), taking place within the Wnt enhanceosome located in the nucleus (blue panel). By contrast, no strutural information is available for the complex between Wnt and its negative regulators Wnt inhibitory factor and secreted-Frizzled related proteins (Figure 3 . red panel in the inset, see WIF1 and SFRP3 with self-edges only). 
bioRxiv preprint doi: https://doi.org/10.1101/2021.07.31.453756; this version posted August 2, 2021. The copyright holder for this preprint (which was not certified by peer review) is the author/funder, who has granted bioRxiv a license to display the preprint in perpetuity. It is made available under aCC-BY-NC-ND 4.0 International license.

LEVELNET: DIVING INTO THE MULTIPLE LAYERS OF PROTEIN-PROTEIN PHYSICAL INTERACTION NETWORKS

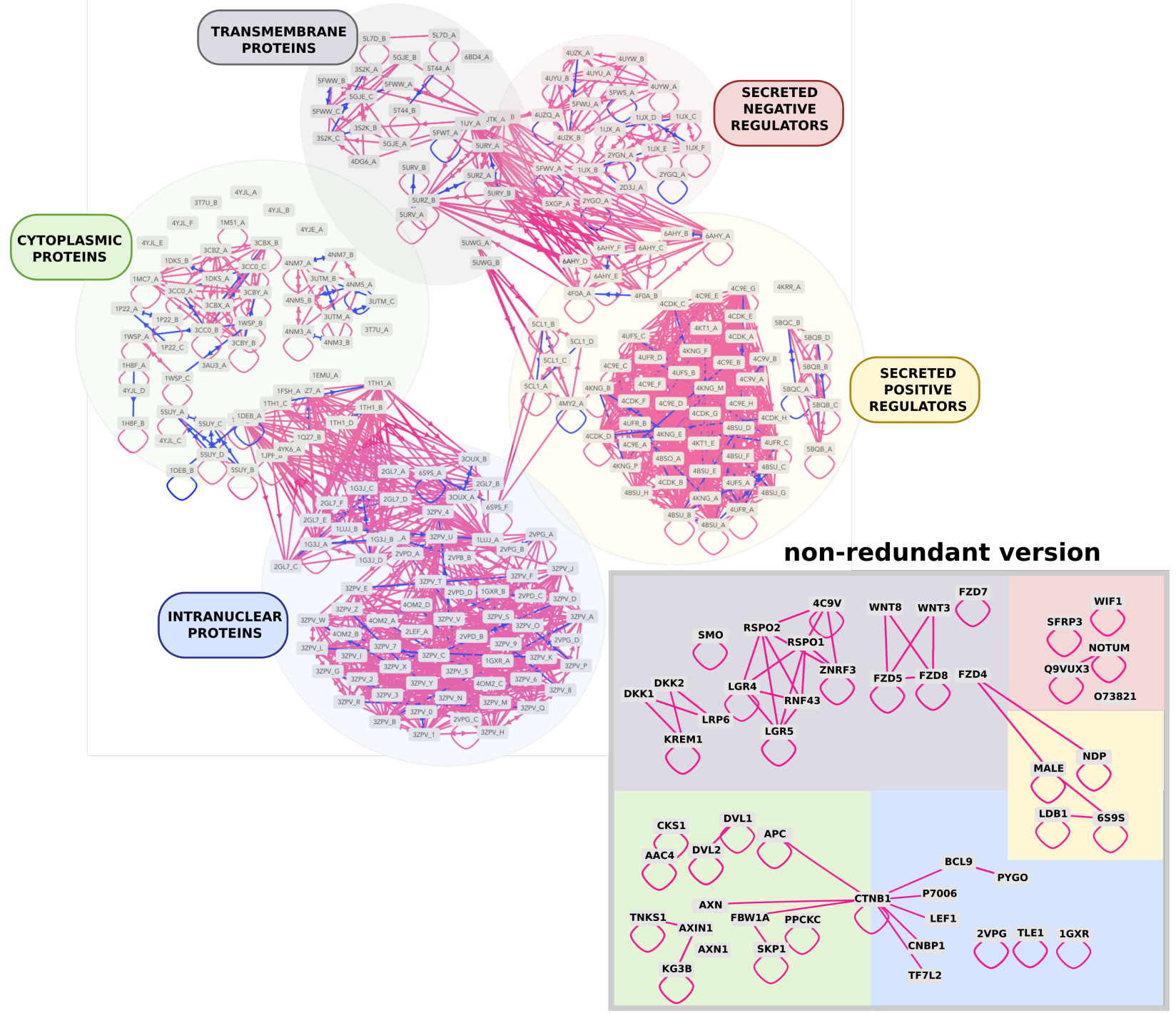

Figure 3: Network inferred for the canonical Wnt signaling pathway. The input PDB codes were taken from (1), and we show the layer corresponding to the interactions inferred from the PDB at 30\% sequence identity. The co-localization annotations of the proteins taken from (1) were given as part of the input, and LEVELNET automatically determined the spatial arrangement of the nodes based on these annotations. The groups corresponding to the different protein cellular locations and roles are labelled. The colors of the circles around the labels are also used in the inset. 
bioRxiv preprint doi: https://doi.org/10.1101/2021.07.31.453756; this version posted August 2, 2021. The copyright holder for this preprint (which was not certified by peer review) is the author/funder, who has granted bioRxiv a license to display the preprint in perpetuity. It is made available under aCC-BY-NC-ND 4.0 International license.

\section{LEVELNET: DIVING INTO THE MULTIPLE LAYERS OF PROTEIN-PROTEIN PHYSICAL INTERACTION NETWORKS}
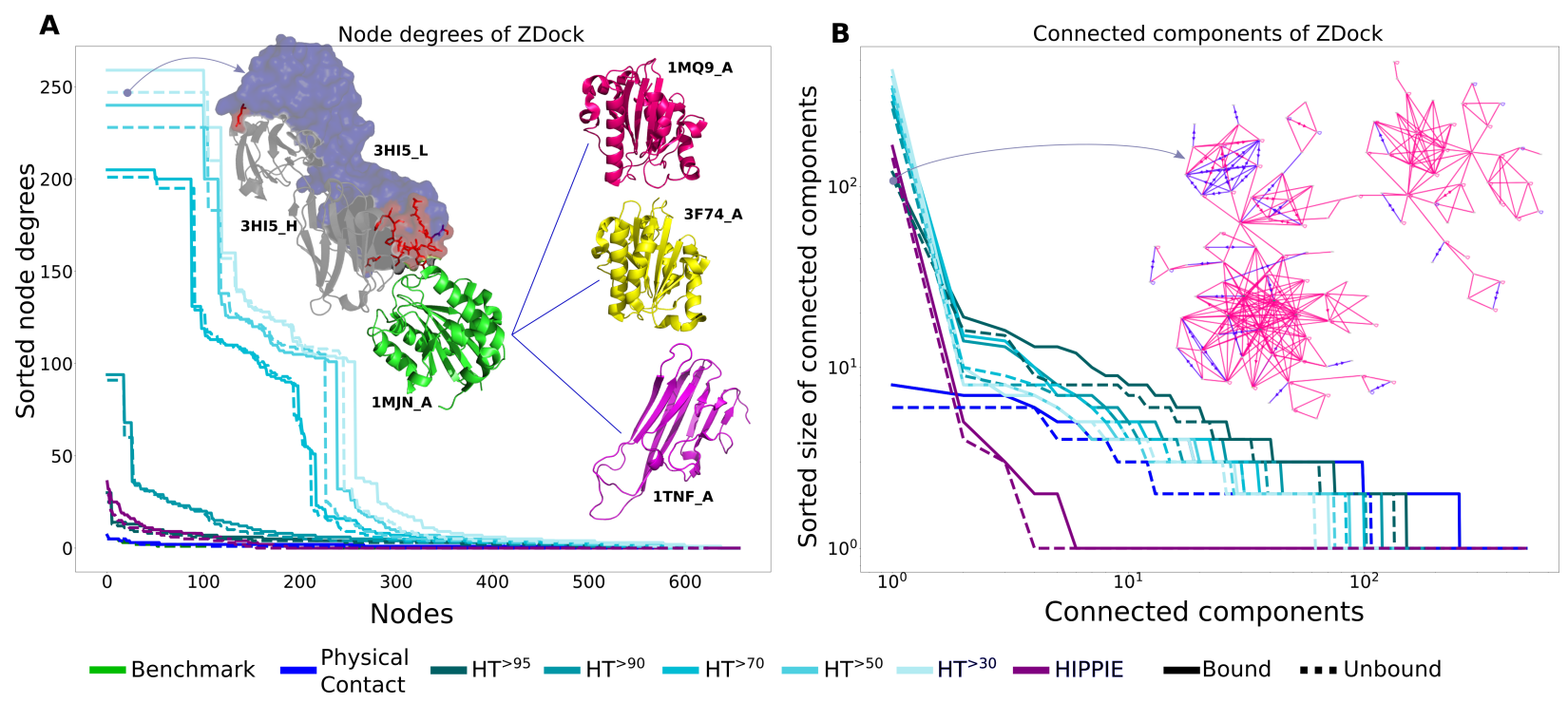

Figure 4: Node degrees and size of connected components in LEVELNET for ZDockv5 database. For each network, the node degrees (A) and the size of connected components (B) are sorted from the largest to the smallest. The $\mathrm{x}$ and $\mathrm{y}$ axis of the connected components are in logarithmic scale. (A) The inset of the plot shows the human FAB structure of 3HI5, characterized by the highest node degree in the dataset. Residues with very high variations among the homologs of this FAB light chain are distinguished by red sticks. The majority of these residues belong to the paratope part of the light chain. By homology transfer at 95\%, this FAB light chain has the potential to interact with other FAB heavy chains or antigens in the dataset, including 1MQ9, 3F74, and the Lymphokines trimer 1TNF. (B) The inset of the plot shows the topology of the largest connected component, obtained at more than $95 \%$ similarity, including the FAB structure of $3 \mathrm{HI} 5$.

\subsection{Assessing PPI predictions}

We used LEVELNET to evaluate the positive PPI predictions obtained from a complete cross-docking experiment reported in (21) performed on the 168 proteins from the ZDock version 2 (ZDockv2) benchmark (22) (Figure 5A). 84 binary interactions are annotated in the benchmark for these proteins. It is important to note that some of the proteins are comprised of several chains. To analyze these predictions, we mapped PPI annotations between protein complexes to annotations between protein chains. Interacting chains in each complex are annotated by 1 and those between two partners are assigned the predicted score. Among the annotated interactions with high confidence (NII > $=0.7), 108$ are backed up by structural data in PDB (Figure 5 A, homology transfer at 95\% sequence identity).

We also assessed the predicted PPI results by considering only 88 single-chain proteins of ZDockv2 (Figure 5B). Out of 3916 pair of chains, the prediction algorithm found 2672 non-interacting ones (Figure $5 \mathrm{~B}$, solid green curve, $N I I>=0.3)$. For the 1244 interactions at confidence score $N I I>=0.379$ and 63 are approved by homology transfers at $70 \%$ and $95 \%$ sequence identities and 37 are approved by physical contacts. Among the 151 predicted interactions with confidence of $N I I>=0.728$ and 19 are approved by homology transfer at $95 \%$ sequence identity and physical contacts, respectively. To further illustrate this application, we considered a subnetwork of predicted interactions reported in (19) comprised of the complex PDB structures 1A2K, 1IBR, 1I2M, and 1K5D from the docking benchmark ZDock version 5.5 (ZDockv5) (40) (Figure 5C). While ZDockv5 annotates only the interactions observed within each PDB complex (blue edges), LEVELNET infers a dense network at 95\% sequence identity (pink edges). This multiplicity of interactions is due to the presence of human Ran GTPase in each structure (node 1A2K_C and nodes in green). 15 predicted interactions (edges in green) are supported by either physical contacts or inferred interactions by homology. One of the predicted interactions, however, is in conflict with the Negatome layer (black edge, chains BC of 1K5D).

\subsection{Unraveling cross-interactions}

We systematically investigated the homology-based inference of cross-interactions within two docking benchmarks, namely ZDockv5 (Figures 4 and S1AC) and DockGround (DG4) (17) (Figure S1BD). Within both benchmarks, some of the protein chains have more than 200 partners at 70\% sequence identity. In case of ZDockv5, the nodes with the 
bioRxiv preprint doi: https://doi.org/10.1101/2021.07.31.453756; this version posted August 2, 2021. The copyright holder for this preprint (which was not certified by peer review) is the author/funder, who has granted bioRxiv a license to display the preprint in perpetuity. It is made available under aCC-BY-NC-ND 4.0 International license.

\section{LEVELNET: DIVING INTO THE MULTIPLE LAYERS OF PROTEIN-PROTEIN PHYSICAL INTERACTION NETWORKS}

A

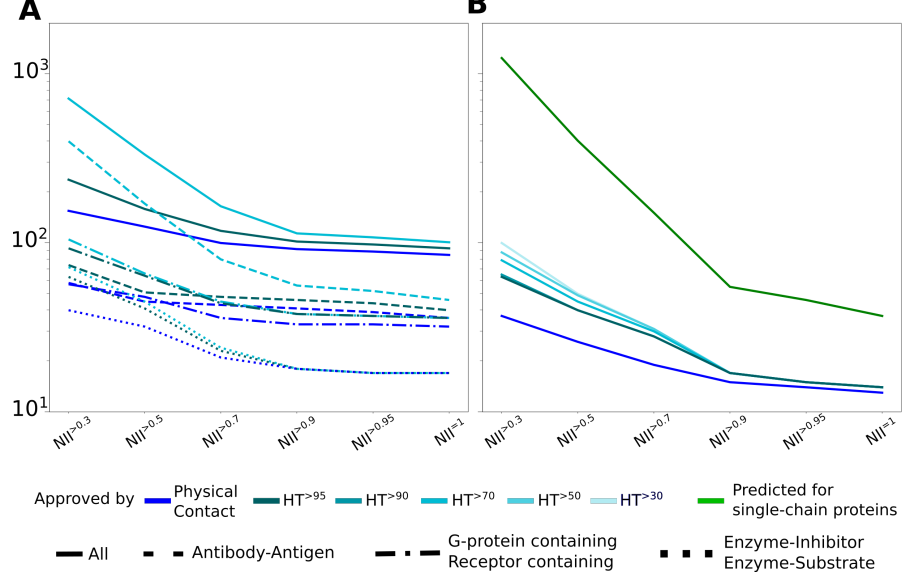

C

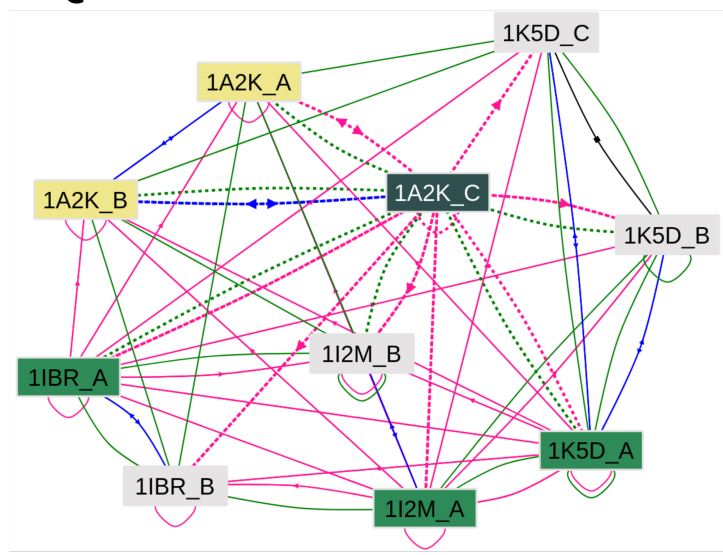

Figure 5: Assessments of PPI predictions. (A-B) An assessment of PPI interactions predicted by an analysis of cross-docking simulations and evolutionary information (21) on ZDockv2. Normalized Interaction Index (NII) is a user-defined confidence score assigned to each interaction. The y axis are in logarithmic scale. (A) A comparison for the whole ZDockv2 and functional classes. (B) An assessment of the predicted PPI results for subset of ZDockv2 including only single-chain proteins. Many predicted interactions that are not annotated in the docking benchmark can be considered as true positives. (C) A subnetwork comparison of docking results for a Ran GTPase and its partners by LEVELNET. NII scores were taken from (19). It assesses the inferred interactions between complexes 1A2K, 1IBR, $1 \mathrm{I} 2 \mathrm{M}$, and $1 \mathrm{~K} 5 \mathrm{D}$ with confidence score more than $40 \%$. By clicking on chain $\mathrm{C}$ of $1 \mathrm{~A} 2 \mathrm{~K}$, its interactions, homologous chains, and other chains of the complex are highlighted.

Table 1: Comparison between PPI resources

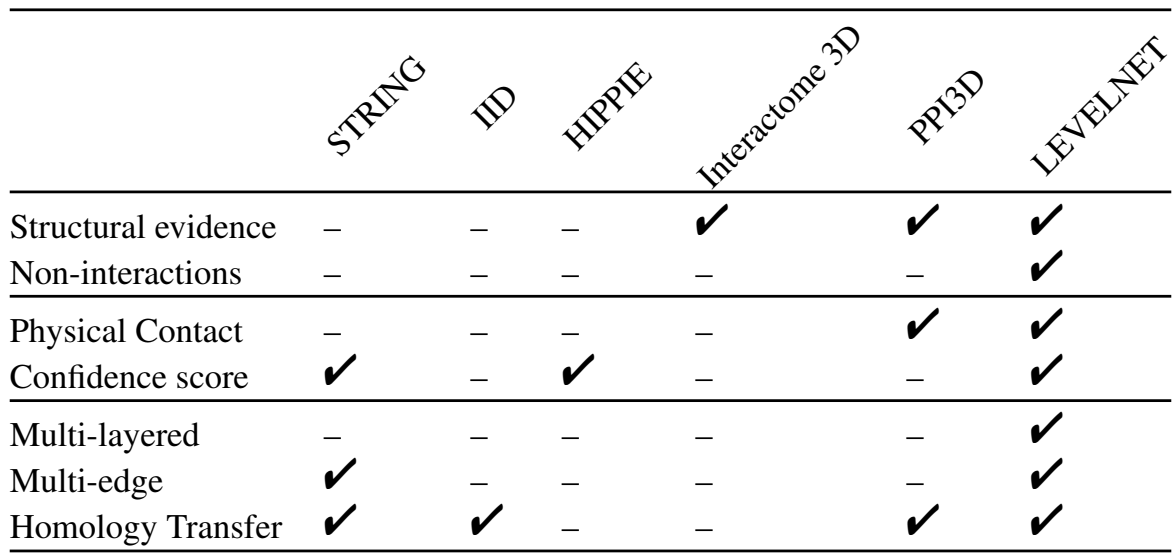

Only HIPPIE focus exclusively on Human and experimental PPI data.

highest degrees correspond to antigen-binding fragments (FAB) (Figure 44). This result reflects the very high sequence similarity shared by FABs recognizing different antigens. Indeed, only a few highly variable residues on antigen-binding site forming the paratope are responsible for the specific recognition of the antigen (Figure $4 \mathrm{~A}$, red sticks). In such cases, transferring interactions by homology may not be valid, even at $95 \%$ sequence identity.

Leaving out this functional class (antibody-antigen) from ZDockv5, we still observe many inferred cross-interactions, with up to 40 partners for one single protein (Figure S1A). The biggest connected components comprise several hundreds of proteins (Figures $4 \mathrm{~B}$ and $\mathrm{S} 1 \mathrm{C}, \mathrm{D}$ ). This type of analyses helps to get a broader view of a set of proteins, beyond the annotations at hand, and also emphasizes the complexity underlying the behaviour of a protein within a community.

Similar considerations hold true for DG4 analysis (Figure $\mathrm{S1} B \mathrm{BD}$ ). 
LEVELNET: DIVING INTO THE MULTIPLE LAYERS OF PROTEIN-PROTEIN PHYSICAL INTERACTION NETWORKS

\subsection{Creating new benchmarks with specific properties}

To showcase the power of LEVELNET, we created a new benchmark set that could be used for assessing docking algorithms. We first selected a set of 500 high-quality hetero-trimeric structures from the PDB (see Methods), which we gave as input to LEVELNET. The resulting network comprises 1206 chains and is organized into 124 connected components at $70 \%$ sequence identity. By merging the homologous nodes at this level, we are left with 112 connected components, among which 45 comprise 3 nodes (represented by 3 master chains). This analysis shows that it is straightforward to compile new benchmarks for assessing specific PPI-related task, like predicting how 3 proteins assemble together. Our procedure guarantees that no cross-interaction in the set exists (based on the available structural information) and that the proteins differ by more than $30 \%$ from each other. The full list of chains from the set is given in the Supplementary Material. The PPI network of this benchmark is shown in Supplementary Figure S4

\subsection{Comparison with other PPI resources}

LEVELNET multi-layered formulation makes it fundamentally different from other PPI resources (Table 1). Moreover, contrary to most resources, it relies primarily on protein structural data, which are growing at a very rapid pace with the cryo-EM revolution. Compared to other structure-based resources like Interactome3D and PPI3D, it has the advantage of including evidence supporting non-interactions, in addition to interactions. Like LEVELNET, Interactome3D allows visualising PPIs in the form of interactive networks, but it does not provide any confidence score or homology transfer. PPI3D provides detailed information about interactions and interfaces but in the form of tables, without a perceptual interactive visualisation of PPI networks. It infers interactions by homology through PSI-BLAST queries, which results into a long response time. LEVELNET, on the other hand, processes user queries very rapidly by pre-building the interaction and physical contact databases.

\section{Conclusion}

Fast and accurate, LEVELNET is a valuable asset for the community to explore protein interactions. It is useful for the biologists interested in the physical contacts of a particular protein or a set of proteins as well as for those who develop and assess computational predictive approaches for interface, partner and complex predictions. It provides a convenient mean to account for different types of relationships between proteins, e.g. functional annotations, cell co-localization, spatiotemporal proteomics, or co-occurrences in publications, and investigate how the latter are correlated to physical interactions. When using this environment, the user must take into account the functional class of the partners when transferring interactions. Future developments will concern the integration of other experimental databases, node selection and merging based on annotations from Uniprot, SCOP or CATH, support of multi-chain proteins and protein-nucleic acid interactions. Future extensions will include more structural information such as description of protein binding sites, information on structural plasticity upon binding, binding affinity, and the effect of mutations over the network. In addition, we want to improve LEVELNET to efficiently process and represent queries for thousands of proteins.

\section{Availability}

LEVELNET is freely available to the community at http://www.lcqb.upmc.fr/levelnet/ All datasets used and generated in this study are made available to the community at http://www.lcqb.upmc.fr/levelnet/\#/tutorial

\section{Conflict of interest statement.}

None declared.

\section{References}

[1] Mark Agostino and Sebastian Öther-Gee Pohl. The structural biology of canonical Wnt signalling. Biochemical Society Transactions, 48(4):1765-1780, July 2020.

[2] Gregorio Alanis-Lobato, Miguel A. Andrade-Navarro, and Martin H. Schaefer. HIPPIE v2.0: enhancing meaningfulness and reliability of protein-protein interaction networks. Nucleic Acids Research, 45(D1):D408-D414, January 2017. Publisher: Oxford Academic. 
LEVELNET: DIVING INTO THE MULTIPLE LAYERS OF PROTEIN-PROTEIN PHYSICAL INTERACTION NETWORKS

[3] Helen Berman, Kim Henrick, Haruki Nakamura, and John L. Markley. The worldwide Protein Data Bank (wwPDB): ensuring a single, uniform archive of PDB data. Nucleic Acids Research, 35(suppl_1):D301-D303, January 2007.

[4] M. Bostock, V. Ogievetsky, and J. Heer. D Data-Driven Documents. IEEE Transactions on Visualization and Computer Graphics, 17(12):2301-2309, December 2011. Conference Name: IEEE Transactions on Visualization and Computer Graphics.

[5] Alberto Calderone, Luisa Castagnoli, and Gianni Cesareni. mentha: a resource for browsing integrated proteininteraction networks. Nature Methods, 10(8):690-691, August 2013. Number: 8 Publisher: Nature Publishing Group.

[6] Justas Dapkūnas, Albertas Timinskas, Kliment Olechnovič, Mindaugas Margelevičius, Rytis Dičiūnas, and Česlovas Venclovas. The PPI3D web server for searching, analyzing and modeling protein-protein interactions in the context of 3D structures. Bioinformatics, 33(6):935-937, March 2017.

[7] Chloé Dequeker, Elodie Laine, and Alessandra Carbone. INTerface Builder: A Fast Protein-Protein Interface Reconstruction Tool. Journal of Chemical Information and Modeling, 57(11):2613-2617, November 2017. Publisher: American Chemical Society.

[8] Chloé Dequeker, Elodie Laine, and Alessandra Carbone. Decrypting protein surfaces by combining evolution, geometry, and molecular docking. Proteins: Structure, Function, and Bioinformatics, 87(11):952-965, 2019.

[9] Max Franz, Harold Rodriguez, Christian Lopes, Khalid Zuberi, Jason Montojo, Gary D Bader, and Quaid Morris. GeneMANIA update 2018. Nucleic Acids Research, 46(W1):W60-W64, July 2018.

[10] Nicolas Guex and Manuel C. Peitsch. SWISS-MODEL and the Swiss-Pdb Viewer: An environment for comparative protein modeling. ELECTROPHORESIS, 18(15):2714-2723, 1997.

[11] G. Traver Hart, Arun K. Ramani, and Edward M. Marcotte. How complete are current yeast and human protein-interaction networks? Genome Biology, 7(11):120, December 2006.

[12] Sohyun Hwang, Chan Yeong Kim, Sunmo Yang, Eiru Kim, Traver Hart, Edward M Marcotte, and Insuk Lee. HumanNet v2: human gene networks for disease research. Nucleic Acids Research, 47(D1):D573-D580, January 2019.

[13] Zerrin Isik, Christoph Baldow, Carlo Vittorio Cannistraci, and Michael Schroeder. Drug target prioritization by perturbed gene expression and network information. Scientific Reports, 5(1):17417, November 2015. Number: 1 Publisher: Nature Publishing Group.

[14] Samuel Kerrien, Bruno Aranda, Lionel Breuza, Alan Bridge, Fiona Broackes-Carter, Carol Chen, Margaret Duesbury, Marine Dumousseau, Marc Feuermann, Ursula Hinz, Christine Jandrasits, Rafael C. Jimenez, Jyoti Khadake, Usha Mahadevan, Patrick Masson, Ivo Pedruzzi, Eric Pfeiffenberger, Pablo Porras, Arathi Raghunath, Bernd Roechert, Sandra Orchard, and Henning Hermjakob. The IntAct molecular interaction database in 2012. Nucleic Acids Research, 40(D1):D841-D846, January 2012.

[15] Max Kotlyar, Chiara Pastrello, Zara Malik, and Igor Jurisica. IID 2018 update: context-specific physical protein-protein interactions in human, model organisms and domesticated species. Nucleic Acids Research, 47(D1):D581-D589, January 2019.

[16] Max Kotlyar, Chiara Pastrello, Nicholas Sheahan, and Igor Jurisica. Integrated interactions database: tissuespecific view of the human and model organism interactomes. Nucleic Acids Research, 44(D1):D536-D541, January 2016.

[17] Petras J. Kundrotas, Ivan Anishchenko, Taras Dauzhenka, Ian Kotthoff, Daniil Mnevets, Matthew M. Copeland, and Ilya A. Vakser. Dockground: A comprehensive data resource for modeling of protein complexes. Protein Science, 27(1):172-181, 2018. _eprint: https://onlinelibrary.wiley.com/doi/pdf/10.1002/pro.3295.

[18] Elodie Laine and Alessandra Carbone. Local Geometry and Evolutionary Conservation of Protein Surfaces Reveal the Multiple Recognition Patches in Protein-Protein Interactions. PLOS Computational Biology, 11(12):e1004580, 2015. Publisher: Public Library of Science.

[19] Elodie Laine and Alessandra Carbone. Protein social behavior makes a stronger signal for partner identification than surface geometry. Proteins, 85(1):137-154, 2017. 
LEVELNET: DIVING INTO THE MULTIPLE LAYERS OF PROTEIN-PROTEIN PHYSICAL INTERACTION NETWORKS

[20] Xian Liu, Cheng Chang, Mingfei Han, Ronghua Yin, Yiqun Zhan, Changyan Li, Changhui Ge, Miao Yu, and Xiaoming Yang. PPIExp: A Web-Based Platform for Integration and Visualization of Protein-Protein Interaction Data and Spatiotemporal Proteomics Data. Journal of Proteome Research, 18(2):633-641, February 2019. Publisher: American Chemical Society.

[21] Anne Lopes, Sophie Sacquin-Mora, Viktoriya Dimitrova, Elodie Laine, Yann Ponty, and Alessandra Carbone. Protein-Protein Interactions in a Crowded Environment: An Analysis via Cross-Docking Simulations and Evolutionary Information. PLOS Computational Biology, 9(12):e1003369, December 2013.

[22] Julian Mintseris, Kevin Wiehe, Brian Pierce, Robert Anderson, Rong Chen, Joël Janin, and Zhiping Weng. Protein-protein docking benchmark 2.0: An update. Proteins: Structure, Function, and Bioinformatics, 60(2):214216, 2005. _eprint: https://onlinelibrary.wiley.com/doi/pdf/10.1002/prot.20560.

[23] Roberto Mosca, Arnaud Céol, and Patrick Aloy. Interactome3D: adding structural details to protein networks. Nature Methods, 10(1):47-53, January 2013. Number: 1 Publisher: Nature Publishing Group.

[24] Roberto Mosca, Arnaud Céol, Amelie Stein, Roger Olivella, and Patrick Aloy. 3did: a catalog of domain-based interactions of known three-dimensional structure. Nucleic Acids Research, 42(D1):D374-D379, January 2014.

[25] Saket Navlakha and Carl Kingsford. The power of protein interaction networks for associating genes with diseases. Bioinformatics, 26(8):1057-1063, April 2010.

[26] Sandra Orchard, Mais Ammari, Bruno Aranda, Lionel Breuza, Leonardo Briganti, Fiona Broackes-Carter, Nancy H. Campbell, Gayatri Chavali, Carol Chen, Noemi del Toro, Margaret Duesbury, Marine Dumousseau, Eugenia Galeota, Ursula Hinz, Marta Iannuccelli, Sruthi Jagannathan, Rafael Jimenez, Jyoti Khadake, Astrid Lagreid, Luana Licata, Ruth C. Lovering, Birgit Meldal, Anna N. Melidoni, Mila Milagros, Daniele Peluso, Livia Perfetto, Pablo Porras, Arathi Raghunath, Sylvie Ricard-Blum, Bernd Roechert, Andre Stutz, Michael Tognolli, Kim van Roey, Gianni Cesareni, and Henning Hermjakob. The MIntAct project-IntAct as a common curation platform for 11 molecular interaction databases. Nucleic Acids Research, 42(D1):D358-D363, January 2014.

[27] Sandra Orchard, Samuel Kerrien, Sara Abbani, Bruno Aranda, Jignesh Bhate, Shelby Bidwell, Alan Bridge, Leonardo Briganti, Fiona S. L. Brinkman, Gianni Cesareni, Andrew Chatr-aryamontri, Emilie Chautard, Carol Chen, Marine Dumousseau, Johannes Goll, Robert E. W. Hancock, Linda I. Hannick, Igor Jurisica, Jyoti Khadake, David J. Lynn, Usha Mahadevan, Livia Perfetto, Arathi Raghunath, Sylvie Ricard-Blum, Bernd Roechert, Lukasz Salwinski, Volker Stümpflen, Mike Tyers, Peter Uetz, Ioannis Xenarios, and Henning Hermjakob. Protein interaction data curation: the International Molecular Exchange (IMEx) consortium. Nature Methods, 9(4):345350, April 2012. Number: 4 Publisher: Nature Publishing Group.

[28] Rose Oughtred, Chris Stark, Bobby-Joe Breitkreutz, Jennifer Rust, Lorrie Boucher, Christie Chang, Nadine Kolas, Lara O'Donnell, Genie Leung, Rochelle McAdam, Frederick Zhang, Sonam Dolma, Andrew Willems, Jasmin Coulombe-Huntington, Andrew Chatr-aryamontri, Kara Dolinski, and Mike Tyers. The BioGRID interaction database: 2019 update. Nucleic Acids Research, 47(D1):D529-D541, January 2019.

[29] L Perfetto, C Pastrello, N del Toro, M Duesbury, M Iannuccelli, M Kotlyar, L Licata, B Meldal, K Panneerselvam, S Panni, N Rahimzadeh, S Ricard-Blum, L Salwinski, A Shrivastava, G Cesareni, M Pellegrini, S Orchard, I Jurisica, H Hermjakob, and P Porras. The IMEx coronavirus interactome: an evolving map of Coronaviridae-host molecular interactions. Database, 2020(baaa096), January 2020.

[30] Janet Piñero, Núria Queralt-Rosinach, Àlex Bravo, Jordi Deu-Pons, Anna Bauer-Mehren, Martin Baron, Ferran Sanz, and Laura I. Furlong. DisGeNET: a discovery platform for the dynamical exploration of human diseases and their genes. Database, 2015(bav028), January 2015.

[31] Pawel Smialowski, Philipp Pagel, Philip Wong, Barbara Brauner, Irmtraud Dunger, Gisela Fobo, Goar Frishman, Corinna Montrone, Thomas Rattei, Dmitrij Frishman, and Andreas Ruepp. The Negatome database: a reference set of non-interacting protein pairs. Nucleic Acids Research, 38(suppl_1):D540-D544, January 2010. Publisher: Oxford Academic.

[32] Martin Steinegger and Johannes Söding. MMseqs2 enables sensitive protein sequence searching for the analysis of massive data sets. Nature Biotechnology, 35(11):1026-1028, November 2017. Number: 11 Publisher: Nature Publishing Group.

[33] Kai Sun, Joana P. Gonçalves, Chris Larminie, and Nataša Pržulj. Predicting disease associations via biological network analysis. BMC Bioinformatics, 15(1):304, September 2014. 
bioRxiv preprint doi: https://doi.org/10.1101/2021.07.31.453756; this version posted August 2, 2021. The copyright holder for this preprint

(which was not certified by peer review) is the author/funder, who has granted bioRxiv a license to display the preprint in perpetuity. It is made available under aCC-BY-NC-ND 4.0 International license.

LEVELNET: DIVING INTO THE MULTIPLE LAYERS OF PROTEIN-PROTEIN PHYSICAL INTERACTION NETWORKS

[34] Damian Szklarczyk, Annika L Gable, David Lyon, Alexander Junge, Stefan Wyder, Jaime Huerta-Cepas, Milan Simonovic, Nadezhda T Doncheva, John H Morris, Peer Bork, Lars J Jensen, and Christian von Mering. STRING v11: protein-protein association networks with increased coverage, supporting functional discovery in genomewide experimental datasets. Nucleic Acids Research, 47(D1):D607-D613, January 2019.

[35] Damian Szklarczyk, Annika L Gable, Katerina C Nastou, David Lyon, Rebecca Kirsch, Sampo Pyysalo, Nadezhda T Doncheva, Marc Legeay, Tao Fang, Peer Bork, Lars J Jensen, and Christian von Mering. The STRING database in 2021: customizable protein-protein networks, and functional characterization of useruploaded gene/measurement sets. Nucleic Acids Research, 49(D1):D605-D612, January 2021.

[36] Damian Szklarczyk, John H Morris, Helen Cook, Michael Kuhn, Stefan Wyder, Milan Simonovic, Alberto Santos, Nadezhda T Doncheva, Alexander Roth, Peer Bork, Lars J. Jensen, and Christian von Mering. The STRING database in 2017: quality-controlled protein-protein association networks, made broadly accessible. Nucleic Acids Research, 45(D1):D362-D368, January 2017.

[37] Xiwei Tang, Qiu Xiao, and Kai Yu. Breast Cancer Candidate Gene Detection Through Integration of Subcellular Localization Data With Protein-Protein Interaction Networks. IEEE Transactions on NanoBioscience, 19(3):556561, July 2020. Conference Name: IEEE Transactions on NanoBioscience.

[38] The UniProt Consortium. UniProt: the universal protein knowledgebase in 2021. Nucleic Acids Research, 49(D1):D480-D489, January 2021.

[39] The UniProt Consortium. UniProt: the universal protein knowledgebase. Nucleic Acids Research, 46(5):2699, March 2018.

[40] Thom Vreven, Iain H. Moal, Anna Vangone, Brian G. Pierce, Panagiotis L. Kastritis, Mieczyslaw Torchala, Raphael Chaleil, Brian Jiménez-García, Paul A. Bates, Juan Fernandez-Recio, Alexandre M. J. J. Bonvin, and Zhiping Weng. Updates to the Integrated Protein-Protein Interaction Benchmarks: Docking Benchmark Version 5 and Affinity Benchmark Version 2. Journal of Molecular Biology, 427(19):3031-3041, September 2015. 
bioRxiv preprint doi: https://doi.org/10.1101/2021.07.31.453756; this version posted August 2, 2021. The copyright holder for this preprint

(which was not certified by peer review) is the author/funder, who has granted bioRxiv a license to display the preprint in perpetuity. It is made available under aCC-BY-NC-ND 4.0 International license.

LEVELNET: DIVING INTO THE MULTIPLE LAYERS OF PROTEIN-PROTEIN PHYSICAL INTERACTION NETWORKS

\section{SUPPLEMENTARY DATA}

Discovery of self- and cross-interactions. The variation of the topology of the networks with respect to the interactions between each layer and corresponding benchmark network is shown in Supplementary Figure S2. Self- and crossinteractions are considered separately and the difference is calculated as the percentage of the maximum possible connections: $n$ and $\left(\begin{array}{l}n \\ 2\end{array}\right)$ (fully connected graph), respectively. The only difference between physical contact and benchmark networks is the self-interactions. These self-interactions are caused by the identical copies of the same chain found in a biological assembly that are in contact. For both benchmarks, more than half of the nodes can interact with themselves at sequence identity above $95 \%$. In case of cross-interactions, ZDockv5 with AA and AS included shows higher differences for both unbound and bound annotations compared to other benchmarks. As a further comparison we studied the pairwise differences in the number of interactions for layers of LEVELNET before and after redundancy reduction (Supplementary Figure S3).

A snapshot of LEVELNET web interface. Supplementary Figure S5 displays a snapshot of the observed and homology transfered interactions with $>95 \%$ sequence identity of single-chain proteins of the ZDock version 5.5 in the bound annotation. The user can highlight and isolate the nodes of the connected protein chains by searching their codes (Supplementary Figure S5D) (the blue connected component subnetwork) as well as follow the trace of homologous chains by clicking on each chain (the node-centered subnetwork) (Supplementary Figure S5A). The essential information of each protein chain will be displayed in a pop-up window by hovering mouse on its node. For a better visualisation the user can control the attractive/repulsive force between the nodes to discriminate clusters of densely connected proteins and also change the edge length, width, strength and node sizes to regulate the dynamic and floating property of the network (Supplementary Figure S5C). The user can visualize another layer of LEVELNET by changing the source, modulating the confidence score, or choosing the non-redundant representation (Supplementary Figure S5B). LEVELNET does not require any plugins; and is compatible with all web browsers. User can download, export, or share the PPI network. LEVELNET is accessible on smartphones and tablets to facilitate the collaboration between researchers. 
LEVELNET: DIVING INTO THE MULTIPLE LAYERS OF PROTEIN-PROTEIN PHYSICAL INTERACTION NETWORKS
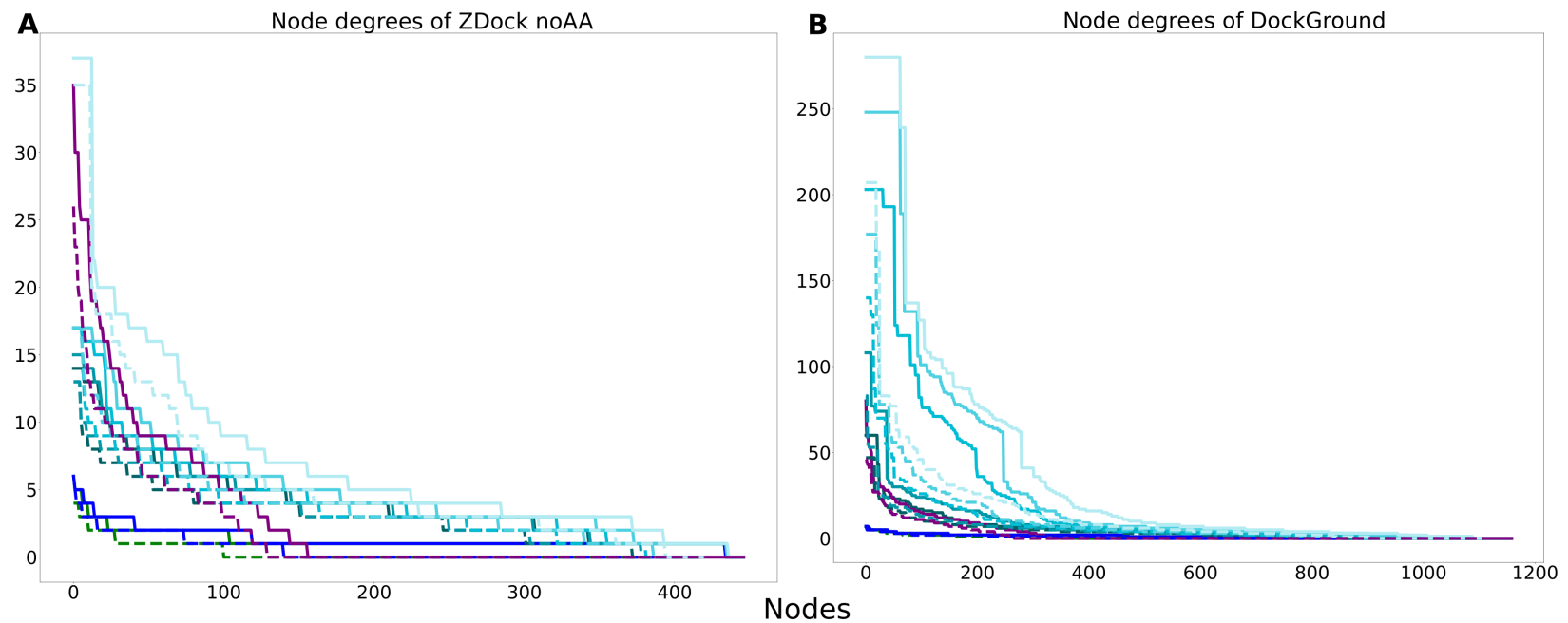

C

Connected components of ZDock noAA

D

Connected components of DockGround
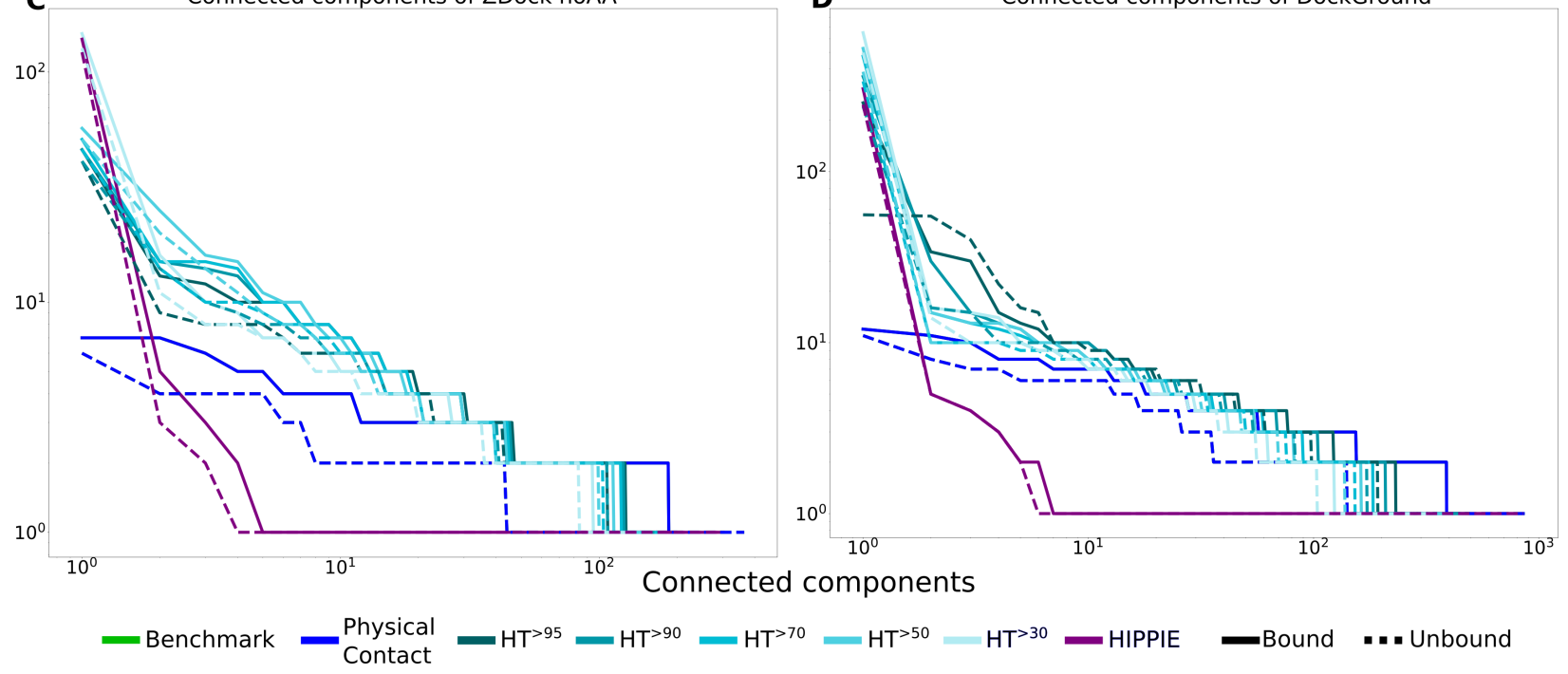

Figure S1: Node degrees and size of connected components in LEVELNET for ZDockv5 (antibody-antigens excluded) and Dockground databases. For each network, whose chains belong to ZDockv5 (AC) and Dockground (BD) databases, the node degrees (AB) and the size of connected components (CD) are sorted from the largest to the smallest. The $\mathrm{x}$ and $\mathrm{y}$ axis of the connected components are in logarithmic scale. 
bioRxiv preprint doi: https://doi.org/10.1101/2021.07.31.453756; this version posted August 2, 2021. The copyright holder for this preprint (which was not certified by peer review) is the author/funder, who has granted bioRxiv a license to display the preprint in perpetuity. It is made available under aCC-BY-NC-ND 4.0 International license.

LEVELNET: DIVING INTO THE MULTIPLE LAYERS OF PROTEIN-PROTEIN PHYSICAL INTERACTION NETWORKS

A

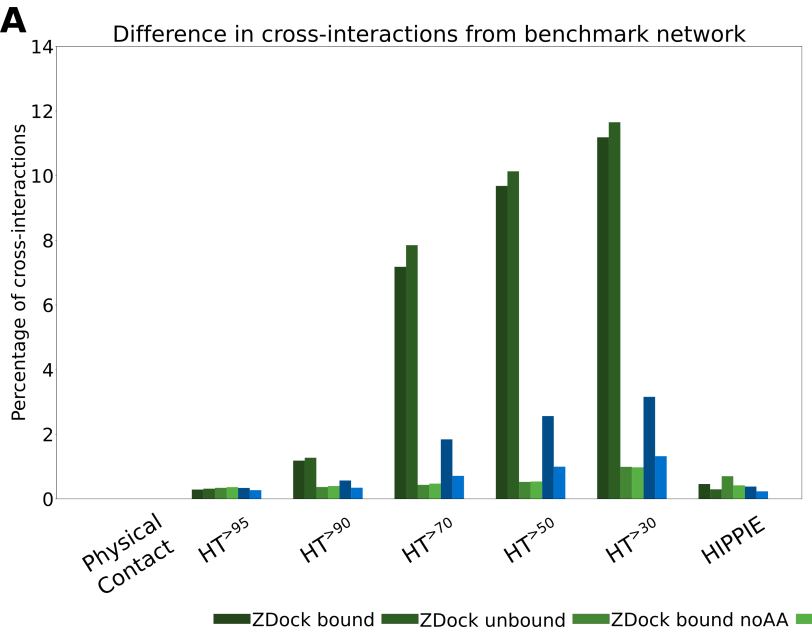

B

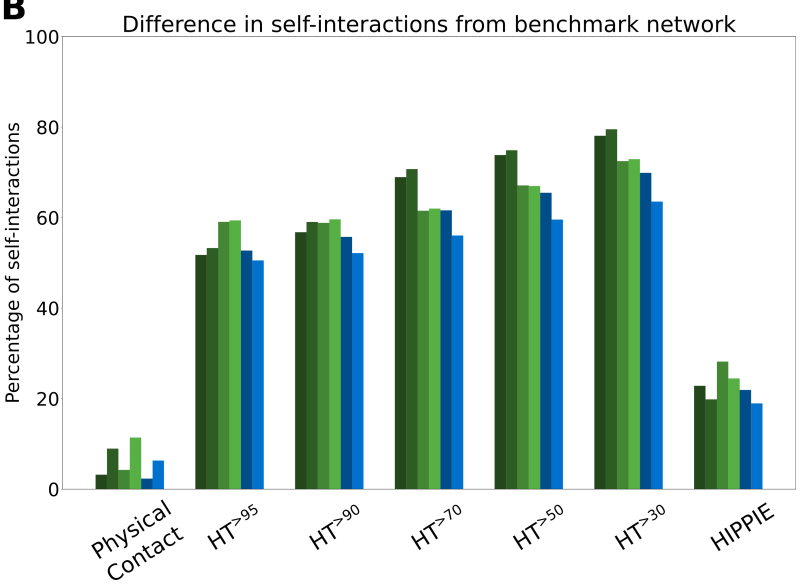

Figure S2: Differences of the interactions in each layer of LEVELNET against the benchmark network (measured in percentage of the maximum possible value). Two types of interactions are considered: (A) self- and (B) crossinteractions with the maximum possible values of $n$ and $\left(\begin{array}{c}n \\ 2\end{array}\right)$ (fully connected graph), respectively.

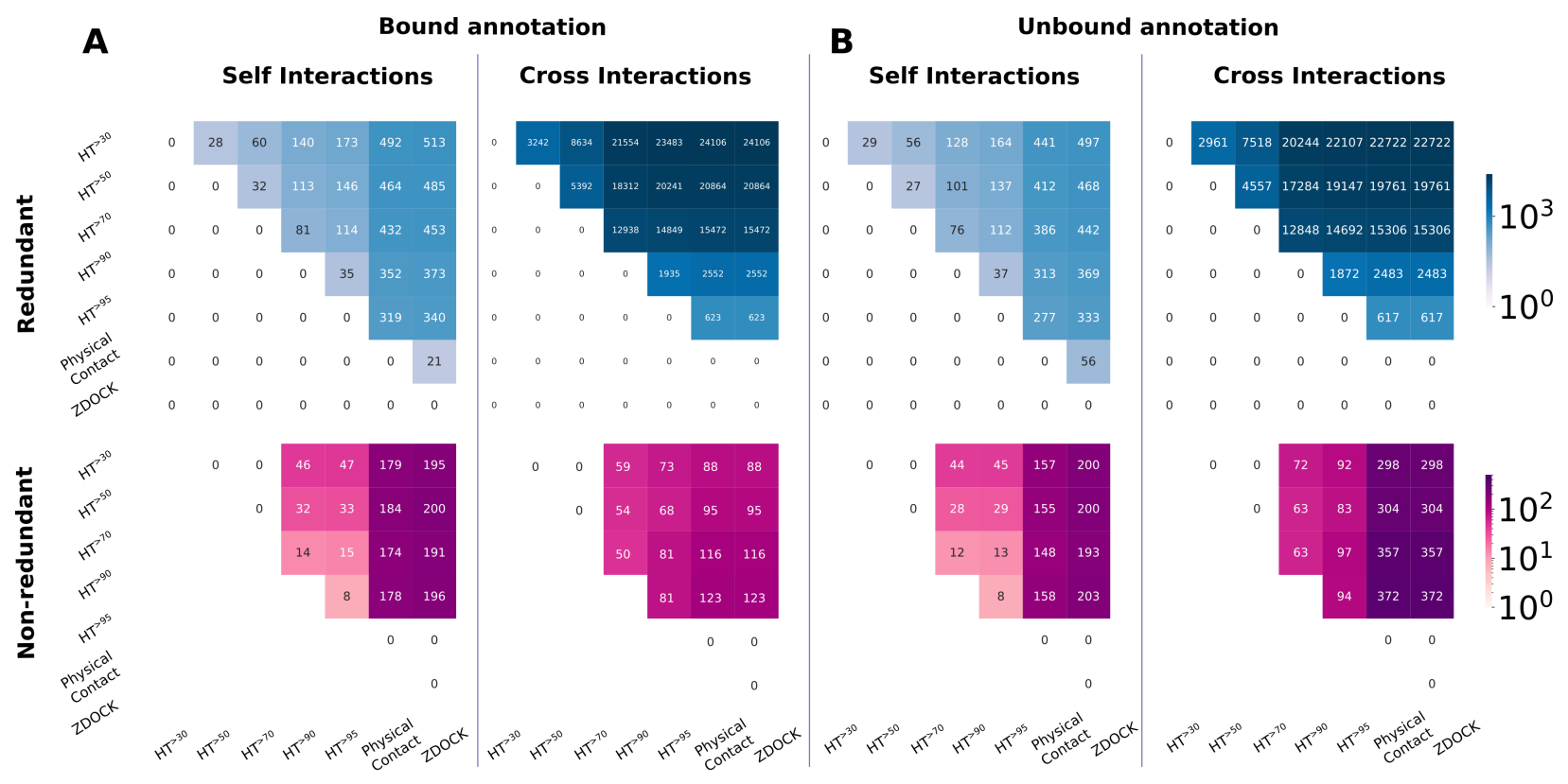

Figure S3: Pairwise differences in the number of interactions for layers of LEVELNET. In the case of redundancy reduction, each network has different set of nodes. In order to compare, for each cell, we took the network with smaller set of nodes (lower sequence similarity) as the reference and compared it to the other network that is built based on the same reference nodes. There is no redundancy reduction for the physical contact and benchmark networks. Two types of interactions are considered: Self- and cross-interactions. (A) ZDockv5 bound annotation. (B) ZDockv5 unbound annotation. 
bioRxiv preprint doi: https://doi.org/10.1101/2021.07.31.453756; this version posted August 2, 2021. The copyright holder for this preprint (which was not certified by peer review) is the author/funder, who has granted bioRxiv a license to display the preprint in perpetuity. It is made available under aCC-BY-NC-ND 4.0 International license.

LEVELNET: DIVING INTO THE MULTIPLE LAYERS OF PROTEIN-PROTEIN PHYSICAL INTERACTION NETWORKS

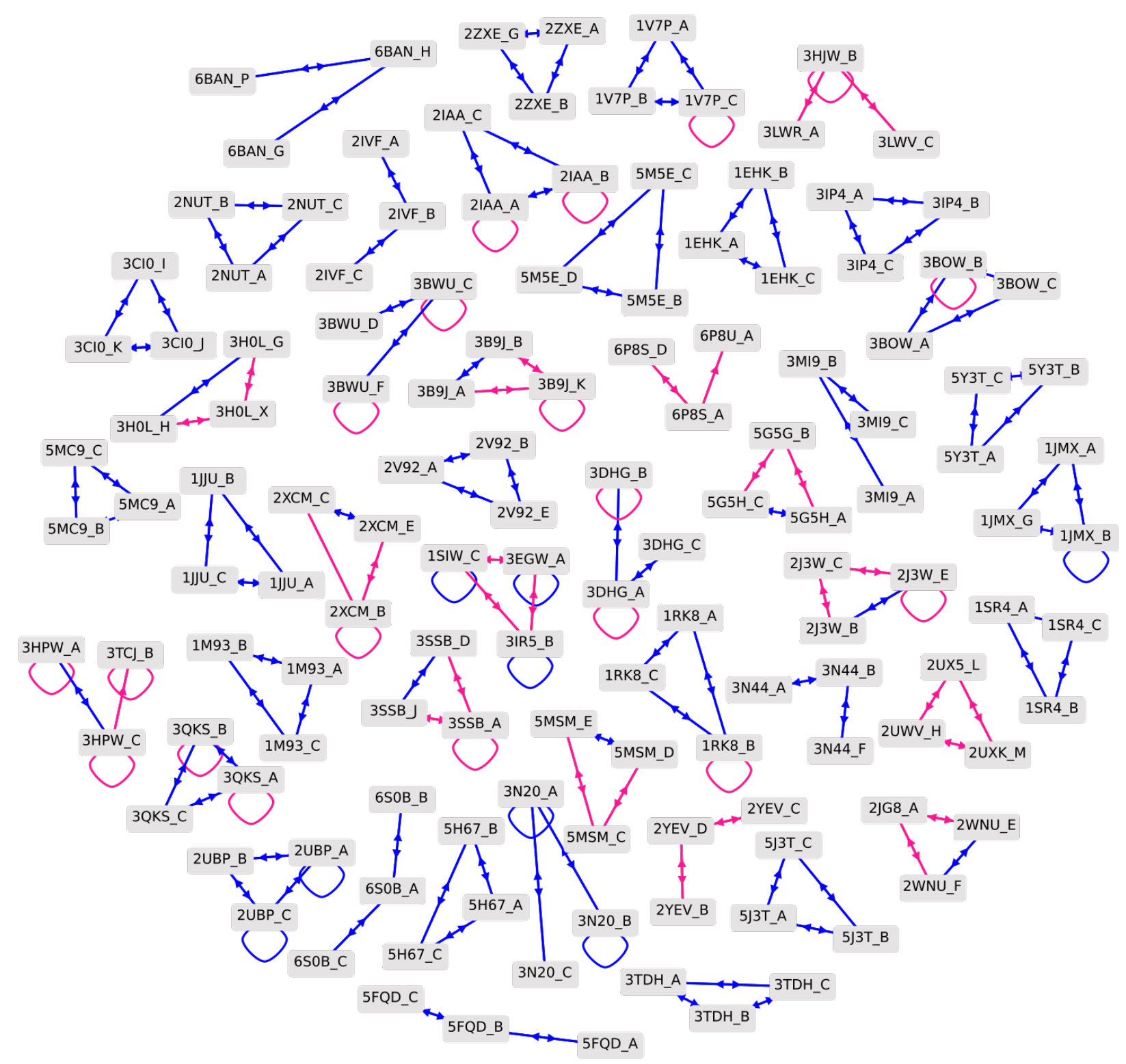

Figure S4: The PPI network of the new benchmark with 45 connected components of size 3 at sequence identity above $70 \%$. 
bioRxiv preprint doi: https://doi.org/10.1101/2021.07.31.453756; this version posted August 2, 2021. The copyright holder for this preprint (which was not certified by peer review) is the author/funder, who has granted bioRxiv a license to display the preprint in perpetuity. It is made available under aCC-BY-NC-ND 4.0 International license.

LEVELNET: DIVING INTO THE MULTIPLE LAYERS OF PROTEIN-PROTEIN PHYSICAL INTERACTION NETWORKS

A
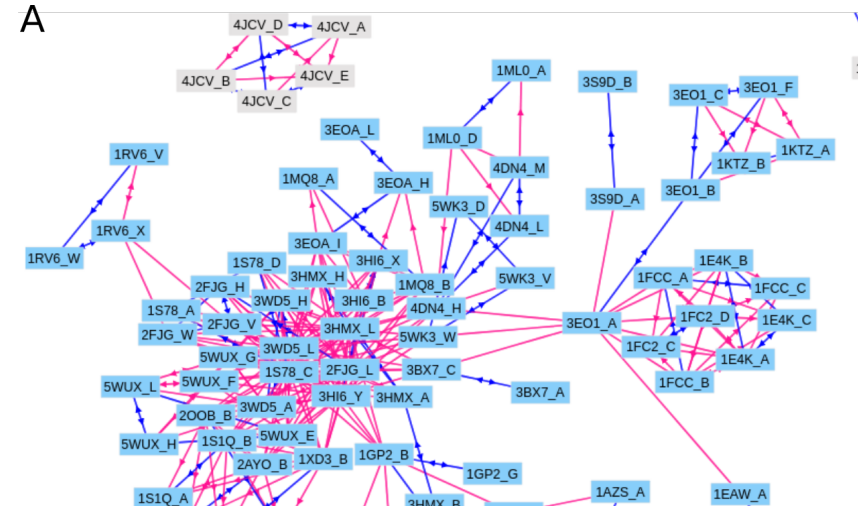

$\perp$ 18WN_p
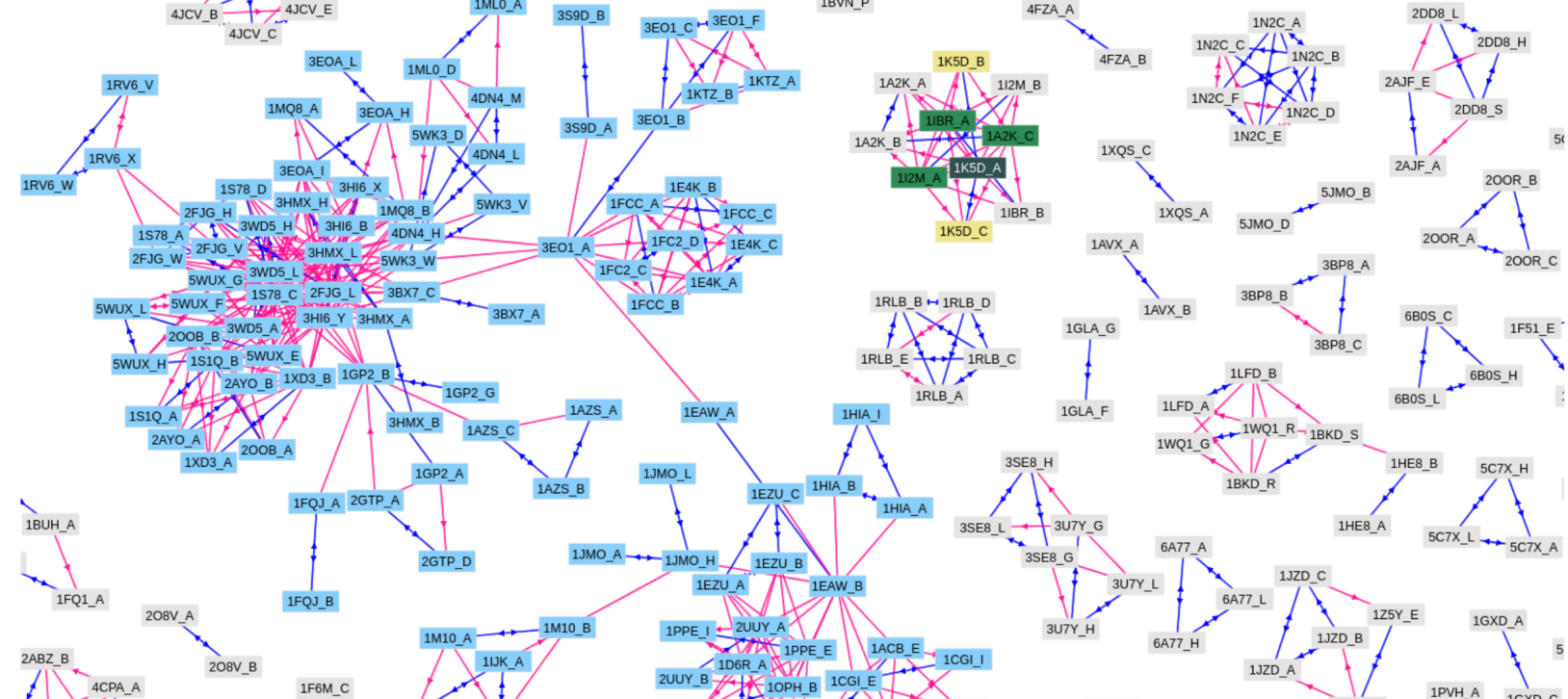

1WQ1_G

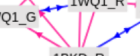

(1)
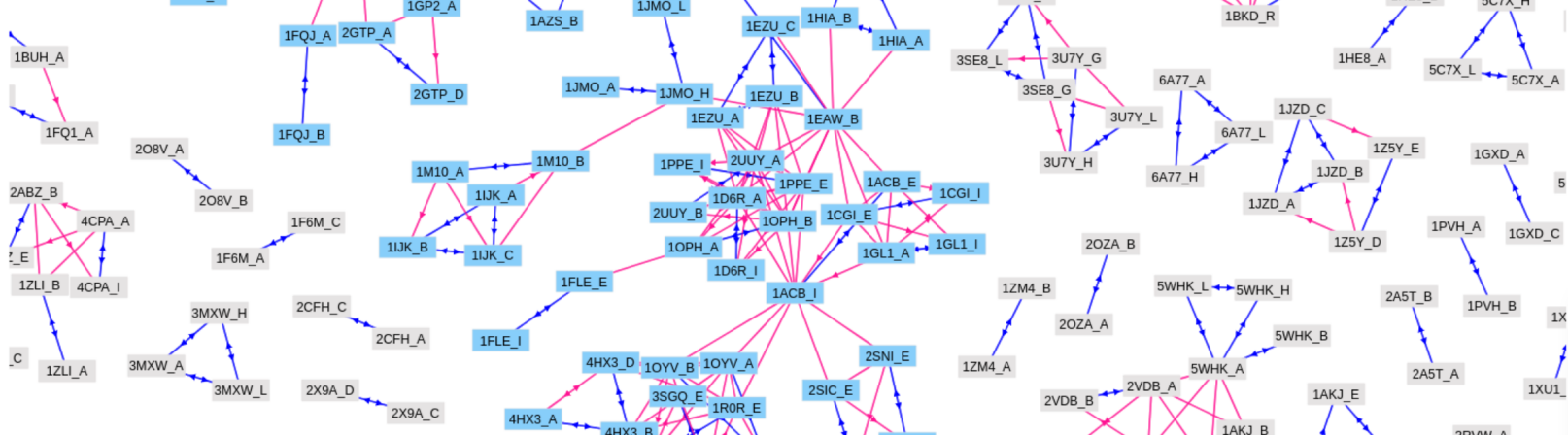

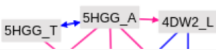

B

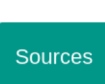

$\bigcirc$ PDB
$\bigcirc$ HIPPIE
$\bigcirc$ User
Negatome
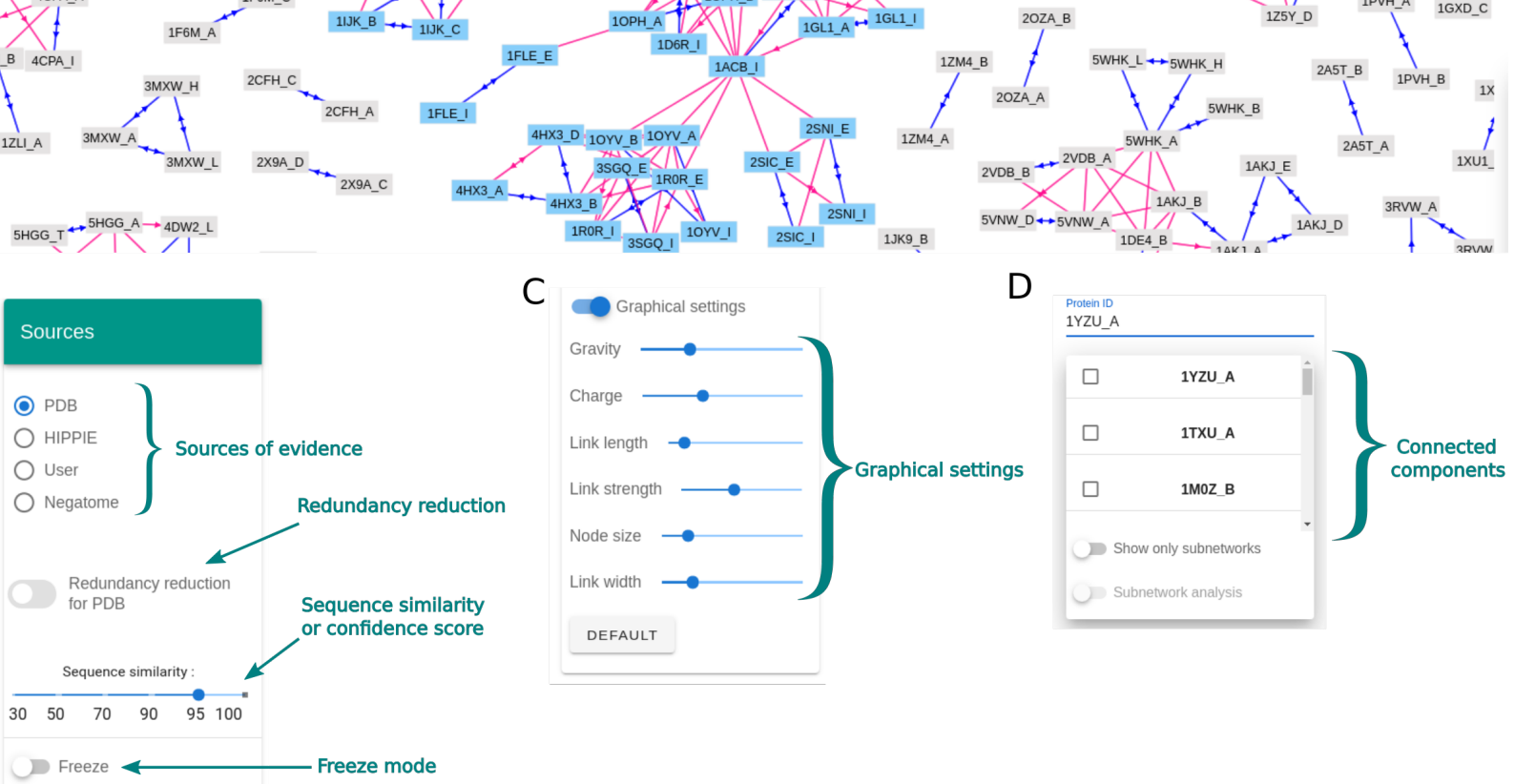

C
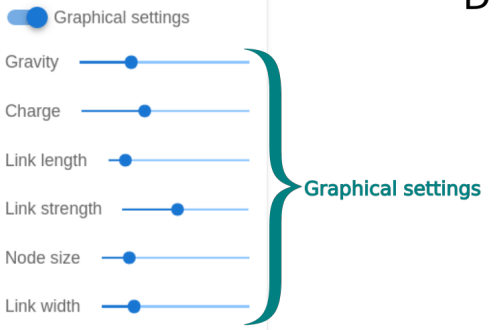

\begin{tabular}{l} 
Protein 10 \\
1YZU_A \\
\hline
\end{tabular}

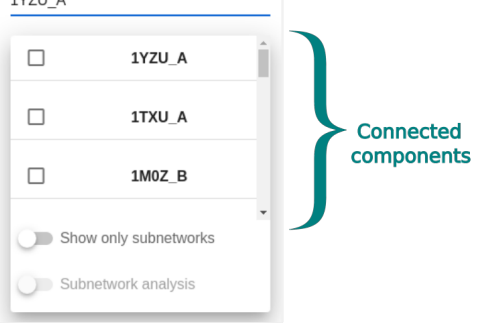

DEFAULT

Figure S5: A snapshot of LEVELNET's web-interface. (A) Observed and inferred interactions with sequence similarity above $95 \%$ of bound single-chain proteins of the ZDockv5. Blue edges represent the existence of observed physical contacts between nodes. Pink edges are inferred by homology propagation from observed evidence. An arrow on an edge means that the partner in the destination is in a bound conformation. (A),(D) The user can search and select a subset of homologous chains or connected components. (B) The user can analyze the network of different layers by either modulating the percentage of sequence similarity or by changing the database of interactions (different confidence scores). (C) Parameters to control the visualisation of the network. 